\title{
$L$-like Combinatorial Principles and Level by Level Equivalence
}

by

\author{
Arthur W. APTER \\ Presented by Czestaw BESSAGA
}

Summary. We force and construct a model in which GCH and level by level equivalence between strong compactness and supercompactness hold, along with certain additional " $L$ like" combinatorial principles. In particular, this model satisfies the following properties:

(1) $\nabla_{\delta}$ holds for every successor and Mahlo cardinal $\delta$.

(2) There is a stationary subset $S$ of the least supercompact cardinal $\kappa_{0}$ such that for every $\delta \in S, \square_{\delta}$ holds and $\delta$ carries a gap 1 morass.

(3) A weak version of $\square_{\delta}$ holds for every infinite cardinal $\delta$.

(4) There is a locally defined well-ordering of the universe $\mathcal{W}$, i.e., for all $\kappa \geq \aleph_{2}$ a regular cardinal, $\mathcal{W}\left\lceil H\left(\kappa^{+}\right)\right.$is definable over the structure $\left\langle H\left(\kappa^{+}\right), \in\right\rangle$ by a parameter free formula.

The model constructed amalgamates and synthesizes results due to the author, the author and Cummings, and Asperó and Sy Friedman. It has no restrictions on the structure of its class of supercompact cardinals and may be considered as part of Friedman's "outer model programme".

1. Introduction and preliminaries. In [11], Sy Friedman introduced the outer model programme, whose goal is to construct "outer models", i.e., forcing extensions, of models of ZFC containing very large cardinals in which $L$-like principles hold. Examples of papers falling under this rubric include, but are not necessarily limited to, [1], [3], [11], [10], [2], [5], [6], 7], and [8].

The purpose of this paper is to produce another contribution to Friedman's outer model programme. We show that the existence of gap 1 morasses on a stationary subset of the least supercompact cardinal $\kappa_{0}$ and a locally

2010 Mathematics Subject Classification: 03E35, 03E55.

Key words and phrases: supercompact cardinal, strongly compact cardinal, strong cardinal, level by level equivalence between strong compactness and supercompactness, diamond, square, morass, locally defined well-ordering. 
defined well-ordering of the universe is consistent with level by level equivalence between strong compactness and supercompactness, together with certain instances of diamond and square. Specifically, we prove the following theorem.

Theorem 1. Suppose $V \vDash " Z F C+G C H+\mathcal{K} \neq \emptyset$ is the class of supercompact cardinals". There is then a partial ordering $\mathbb{P} \subseteq V$ such that $V^{\mathbb{P}} \vDash$ "ZFC $+G C H+\mathcal{K}$ is the class of supercompact cardinals". In $V^{\mathbb{P}}$, level by level equivalence between strong compactness and supercompactness holds. In addition, $V^{\mathbb{P}}$ satisfies the following combinatorial properties:

(1) $\nabla_{\delta}$ holds for every successor and Mahlo cardinal $\delta\left({ }^{1}\right)$.

(2) There is a stationary subset $S$ of the least supercompact cardinal $\kappa_{0}$ such that for every $\delta \in S, \square_{\delta}$ holds and $\delta$ carries a gap 1 morass.

(3) A weak version of $\square_{\delta}$ holds for every infinite cardinal $\delta$. In particular, $\square_{\delta}^{T}$ holds for every infinite cardinal $\delta$, where $T=\operatorname{Safe}(\delta)$ is a certain final segment of regular cardinals less than or equal to $\delta\left({ }^{2}\right)$.

(4) There is a locally defined well-ordering of the universe $\mathcal{W}$, i.e., for all regular cardinals $\kappa \geq \aleph_{2}, \mathcal{W} \backslash H\left(\kappa^{+}\right)$is definable over the structure $\left\langle H\left(\kappa^{+}\right), \in\right\rangle$ by a parameter free formula.

The model witnessing the conclusions of Theorem 1 amalgamates and synthesizes the results of [1], [3, and [5]. Since our ground model has no restrictions on the structure of its class of supercompact cardinals, neither does the resulting generic extension. It is produced by forcing over the model of [3] with a modified version of one of the partial orderings used in [1] (which will add gap 1 morasses and square sequences to each member of a certain stationary subset of the least supercompact cardinal) and the partial ordering of [5]. The key step will be to show that the forcing of [5] preserves the combinatorial properties previously added, together with level by level equivalence between strong compactness and supercompactness.

Before beginning the proof of Theorem 1, we give the most pertinent definitions and facts, many of which are taken from [1] or [3]. When forcing, $q \geq p$ means that $q$ is stronger than $p$. Let $\kappa$ be a cardinal. We abuse notation slightly by using both $V[G]$ and $V^{\mathbb{P}}$ to denote the generic extension of $V$ by $\mathbb{P}$, assuming that $G$ is $V$-generic over $\mathbb{P}$. The partial ordering $\mathbb{P}$ is $\kappa$-directed closed if every directed set of elements of $\mathbb{P}$ of size less than $\kappa$ has an upper bound. $\mathbb{P}$ is $\kappa$-closed if every increasing chain of elements of $\mathbb{P}$ of size less than $\kappa$ has an upper bound. $\mathbb{P}$ is $\kappa$-strategically closed if in the two person game in which the players construct an increasing sequence

$\left({ }^{1}\right)$ Of course, the fact that $\diamond_{\delta}$ holds for every successor cardinal $\delta>\aleph_{1}$ follows from $\mathrm{GCH}$, by Shelah's famous result [17] on diamonds.

$\left({ }^{2}\right)$ The exact definition of $\operatorname{Safe}(\delta)$ will be given in Definition 1.2 
$\left\langle p_{\alpha} \mid \alpha \leq \kappa\right\rangle$ of elements of $\mathbb{P}$, where player I plays odd stages and player II plays even stages (choosing the trivial condition at stage 0), player II has a strategy ensuring the game can always be continued. $\mathbb{P}$ is $\prec \kappa$-strategically closed if in the two person game in which the players construct an increasing sequence $\left\langle p_{\alpha} \mid \alpha<\kappa\right\rangle$ of elements of $\mathbb{P}$, where player I plays odd stages and player II plays even stages (choosing the trivial condition at stage 0), player II has a strategy ensuring the game can always be continued. Note that for any partial ordering $\mathbb{P}$, we have the chain of implications $\kappa$-directed closed $\Rightarrow$ $\kappa$-closed $\Rightarrow \prec \kappa$-strategically closed. In addition, if $\mathbb{P}$ is $\kappa$-strategically closed and $f: \kappa \rightarrow V$ is a function in $V^{\mathbb{P}}$, then $f \in V$.

Next, we state a result which will be used in the proof of Theorem 1. This is a corollary of Theorems 3 and 31 and Corollary 14 of Hamkins' paper [12]. This theorem is a generalization of Hamkins' Gap Forcing Theorem and Corollary 16 of [13] and [14] (and we refer readers to [13], [14], and [12] for further details). We therefore state the theorem we will be using now, along with some associated terminology. Suppose $\mathbb{P}$ is a partial ordering which can be written as $\mathbb{Q} * \dot{\mathbb{R}}$, where $|\mathbb{Q}| \leq \delta, \mathbb{Q}$ is nontrivial, and $\vdash_{\mathbb{Q}}$ " $\mathbb{R}$ is $\delta$-strategically closed". In Hamkins' terminology of [12], $\mathbb{P}$ admits a closure point at $\delta$. In Hamkins' terminology of [13] and [14, $\mathbb{P}$ is mild with respect to a cardinal $\kappa$ iff every set of ordinals $x$ in $V^{\mathbb{P}}$ of size below $\kappa$ has a "nice" name $\tau$ in $V$ of size below $\kappa$, i.e., there is a set $y$ in $V,|y|<\kappa$, such that any ordinal forced by a condition in $\mathbb{P}$ to be in $\tau$ is an element of $y$. Also, as in the terminology of [13, [14, [12, and elsewhere, an embedding $j: \bar{V} \rightarrow \bar{M}$ is amenable to $\bar{V}$ when $j\lceil A \in \bar{V}$ for any $A \in \bar{V}$. The specific corollary of Theorems 3 and 31 and Corollary 14 of [12] we will be using is then the following.

Theorem 2 (Hamkins). Suppose that $V[G]$ is a forcing extension obtained by forcing that admits a closure point at some regular $\delta<\kappa$. Suppose further that $j: V[G] \rightarrow M[j(G)]$ is an embedding with critical point $\kappa$ for which $M[j(G)] \subseteq V[G]$ and $M[j(G)]^{\delta} \subseteq M[j(G)]$ in $V[G]$. Then $M \subseteq V$; indeed, $M=V \cap M[j(G)]$. If the full embedding $j$ is amenable to $V[G]$, then the restricted embedding $j\lceil V: V \rightarrow M$ is amenable to $V$. If $j$ is definable from parameters (such as a measure or extender) in $V[G]$, then the restricted embedding $j\lceil V$ is definable from the names of those parameters in $V$. Finally, for any pair of cardinals $\lambda \geq \kappa$, if $\mathbb{P}$ is mild with respect to $\kappa$ and $\kappa$ is $\lambda$ strongly compact in $V[G]$, then $\kappa$ is $\lambda$ strongly compact in $V$.

It immediately follows from Theorem 2 that any cardinal $\kappa$ which is $\lambda$ supercompact in a generic extension obtained by forcing that admits a closure point below $\kappa$ (such as at or less than the least inaccessible cardinal) must also be $\lambda$ supercompact in the ground model. In particular, if $\bar{V}$ is a forcing extension of $V$ by a partial ordering that admits a closure point at or less 
than the least inaccessible cardinal in which each supercompact cardinal is preserved, the class of supercompact cardinals in $\bar{V}$ remains the same as in $V$.

Suppose $V$ is a model of ZFC in which for all regular cardinals $\kappa<\lambda$, $\kappa$ is $\lambda$ strongly compact iff $\kappa$ is $\lambda$ supercompact, except possibly if $\kappa$ is a measurable limit of cardinals $\delta$ which are $\lambda$ supercompact. Such a model will be said to witness level by level equivalence between strong compactness and supercompactness. Note that the exception is provided by a theorem of Menas [16], who showed that if $\kappa$ is a measurable limit of cardinals $\delta$ which are $\lambda$ strongly compact, then $\kappa$ is $\lambda$ strongly compact but need not be $\lambda$ supercompact. Models in which level by level equivalence between strong compactness and supercompactness holds nontrivially were first constructed in [4].

We presume familiarity with the combinatorial notions of $\square, \diamond$ and its variants, and morasses. We refer readers to [1], [3], and [7] for further information. Since it is somewhat less familiar, however, we do state a weak version of $\square_{\gamma}, \square_{\gamma}^{T}$, compatible with supercompact cardinals. Square sequences of this kind were first shown to be consistent with supercompactness by Foreman and Magidor [9, p. 191], using techniques of Baumgartner. In the notation of Definition 1.1. Foreman and Magidor showed that $\square_{\kappa^{+\omega}}^{\left\{\kappa^{+n} \mid n<\omega\right\}}$ is consistent with $\kappa$ being supercompact.

Given a set $T$ of regular cardinals, we denote by $\operatorname{cof}(T)$ the class of ordinals $\alpha$ such that $\operatorname{cf}(\alpha) \in T$.

Definition 1.1. Let $\gamma$ be an infinite cardinal, and let $T$ be a set of regular cardinals which are less than or equal to $\gamma$. Then a $\square_{\gamma}^{T}$ sequence is a sequence $\left\langle C_{\alpha} \mid \alpha \in \gamma^{+} \cap \operatorname{cof}(T)\right\rangle$ such that

1. $C_{\alpha}$ is club in $\alpha$, and ot $\left(C_{\alpha}\right) \leq \gamma$.

2. If $\beta \in \lim \left(C_{\alpha}\right) \cap \lim \left(C_{\alpha^{\prime}}\right)$, then $C_{\alpha} \cap \beta=C_{\alpha^{\prime}} \cap \beta$.

$\square_{\gamma}^{T}$ holds if and only if there is a $\square_{\gamma}^{T}$ sequence.

Definition 1.2. For each infinite cardinal $\gamma$, a regular cardinal $\mu$ is safe for $\gamma$ if and only if

1. $\mu \leq \gamma$.

2. For every cardinal $\lambda \leq \gamma$, if $\lambda$ is $\gamma^{+}$supercompact, then $\lambda \leq \mu$. $\operatorname{Safe}(\gamma)$ is the set of safe regular cardinals for $\gamma$.

We observe that the safe set is a final segment of $\operatorname{REG} \cap(\gamma+1)$, and that the safe set can only be empty when $\gamma$ is a singular limit of cardinals which are $\gamma^{+}$supercompact. In addition, by the remarks immediately following the statement of Theorem 2, $\operatorname{Safe}(\gamma)$ is upwards absolute to any cofinality (and hence cardinal) preserving generic extension by a partial ordering admitting a closure point at or below the least inaccessible cardinal. 
2. The proof of Theorem 1. Suppose $V \vDash$ "ZFC $+\mathrm{GCH}+\mathcal{K} \neq \emptyset$ is the class of supercompact cardinals". By first forcing with the partial ordering of [3], we assume in addition that level by level equivalence between strong compactness and supercompactness holds in $V$ and that $V \vDash " \nabla_{\delta}$ holds for every regular cardinal $\left.\delta\left[{ }^{3}\right)\right]+\square_{\delta}^{T}$ holds for every infinite cardinal $\delta$, where $T=\operatorname{Safe}(\delta) "$.

Let $\kappa_{0}$ be the least supercompact cardinal, and let $S=\left\{\delta<\kappa_{0} \mid \delta\right.$ is a non-measurable Mahlo limit of strong cardinals $\}$. As in [1, $S$ is stationary. This allows us to define our first partial ordering $\mathbb{P}^{*}=\left\langle\left\langle\mathbb{P}_{\delta}, \dot{\mathbb{Q}}_{\delta}\right\rangle \mid \delta<\kappa_{0}\right\rangle$ as the reverse Easton iteration of length $\kappa_{0}$ which begins by forcing with $\operatorname{Add}\left(\aleph_{1}, 1\right)$, the partial ordering for adding a Cohen subset of $\aleph_{1}$. For any stage $\delta>0, \dot{\mathbb{Q}}_{\delta}$ is a term for the trivial partial ordering $\{\emptyset\}$, except if $\delta \in S$. Under these circumstances, $\dot{\mathbb{Q}}_{\delta}=\dot{\mathbb{Q}}_{\delta}^{0} * \dot{\mathbb{Q}}_{\delta}^{1}$, where $\dot{\mathbb{Q}}_{\delta}^{0}$ is a term for the partial ordering of [1] which adds a $\square_{\delta}$ sequence, and $\dot{\mathbb{Q}}_{\delta}^{1}$ is a term for the partial ordering of [11] (see also [7]) which adds a gap 1 morass at $\delta\left({ }^{4}\right)$. Since $\mathbb{P}^{*}$ is a reverse Easton iteration of length $\kappa_{0}$ and $\kappa_{0}$ is Mahlo, $\mathbb{P}^{*}$ is $\kappa_{0}$-c.c. Hence, it follows that $V^{\mathbb{P}^{*}} \vDash$ " $S$ is a stationary subset of $\kappa_{0}$ ".

Suppose $\delta \in S$. It is inductively the case that forcing with either $\mathbb{P}_{\delta}$ or

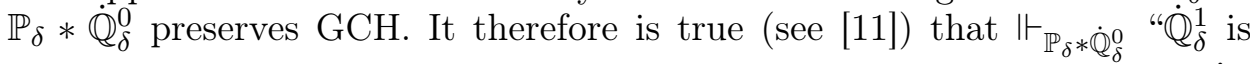
$\delta$-closed and $\delta^{+}$-c.c.". This of course means that $\Vdash_{\mathbb{P}_{\delta} * \dot{\mathbb{Q}}_{\delta}^{0}}$ "Forcing with $\dot{\mathbb{Q}}_{\delta}^{1}$ preserves all cofinalities". It is also the case (because of the way in which $\mathbb{Q}_{\delta}^{1}$ is defined and because $\Vdash_{\mathbb{P}_{\delta} * \dot{\mathbb{Q}}_{\delta}^{0}}$ "这 ${ }_{\delta}^{1}$ is $\delta$-closed and $\delta^{+}$-c.c.") that $\Vdash_{\mathbb{P}_{\delta} * \dot{\mathbb{Q}}_{\delta}^{0}}$ "Forcing with $\dot{\mathbb{Q}}_{\delta}^{1}$ preserves GCH". Because $\square_{\delta}$ is upwards absolute to a cofinality preserving generic extension, we may thus infer that $\Vdash_{\mathbb{P}_{\delta} * \dot{\mathbb{Q}}_{\delta}^{0} * \dot{\mathbb{Q}}_{\delta}^{1}}$ "There is both a $\square_{\delta}$ sequence and a gap 1 morass at $\delta$ ". In addition, since

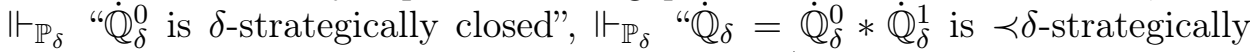
closed". We may consequently conclude that $V^{\mathbb{P}^{*}} \vDash " S$ is a stationary subset of $\kappa_{0}$ such that for every $\delta \in S, \square_{\delta}$ holds and $\delta$ carries a gap 1 morass". Further, $\mathbb{P}^{*}$ admits a closure point at $\aleph_{1}$, and inductively, forcing with $\mathbb{P}^{*}$ preserves cofinalities. Thus, by our remarks immediately following Definition 1.2. $\operatorname{Safe}(\delta)$ is upwards absolute to $V^{\mathbb{P}^{*}}$ for every infinite cardinal $\delta$. This means that $V^{\mathbb{P}^{*}} \vDash " \square \square_{\delta}^{T}$ holds for every infinite cardinal $\delta$, where $T=\operatorname{Safe}(\delta)$ ".

$\left({ }^{3}\right)$ In fact, forcing with the partial ordering of 3] actually allows us to assume that $V \vDash " \diamond_{\delta}^{+}$holds for every $\delta$ which is the successor of a regular cardinal".

$\left({ }^{4}\right)$ Quoting [1] the partial ordering for adding a $\square_{\delta}$ sequence consists of proper initial segments of $\square_{\delta}$ sequences, ordered by end-extension. Quoting [11, the partial ordering for adding a gap 1 morass at $\delta$ consists of proper initial segments of a gap 1 morass at $\delta$ up to some top level, together with a map of an initial segment of this top level into $\delta^{+}$which obeys the requirements of a morass map. The ordering is by end-extending the morass up to its top level and requiring that the map from the given initial segment of its top level into $\delta^{+}$factors as the composition of a map into the top level of the stronger condition, followed by the map given by the stronger condition into $\delta^{+}$. 
By exactly the same arguments as in [1, Lemma 1.7], we have that if $V \vDash$ " $\delta<\lambda$ are such that $\delta$ is $\lambda$ supercompact and $\lambda$ is regular", then $V^{\mathbb{P}^{*}} \vDash$ " $\delta$ is $\lambda$ supercompact" $\left({ }^{5}\right)$. This means that each supercompact cardinal is preserved from $V$ to $V^{\mathbb{P}^{*}}$. Consequently, since $\mathbb{P}^{*}$ admits a closure point at $\aleph_{1}$, by our remarks immediately following the statement of Theorem $2, V^{\mathbb{P}^{*}} \vDash$ " $\mathcal{K}$ is the class of supercompact cardinals (so $\kappa_{0}$ is the least supercompact cardinal)". Further, $V^{\mathbb{P}^{*}} \vDash$ "Level by level equivalence between strong compactness and supercompactness holds", by the identical argument to the one given in the proof of [1, Lemma 1.3] $\left({ }^{6}\right)$

Since an inductive argument together with the facts of the preceding paragraph show that $V^{\mathbb{P}^{*}} \vDash G C H$, Shelah's result of 17 immediately implies that $V^{\mathbb{P}^{*}} \vDash " \nabla_{\delta}$ holds for every successor cardinal $\delta>\aleph_{1}$ ". Because the first nontrivial stage of $\mathbb{P}^{*}$ adds a Cohen subset of $\aleph_{1}$, as in [1, $V^{\mathbb{P}^{*}} \vDash " \nabla \aleph_{1}$ holds", i.e., $V^{\mathbb{P}^{*}} \vDash " \triangle$ holds". Now, for $\delta$ such that $V^{\mathbb{P}^{*}} \vDash " \delta$ is a Mahlo cardinal", it is the case that $V \vDash$ " $\delta$ is a Mahlo cardinal". If we write $\mathbb{P}^{*}=\mathbb{P}_{\delta} * \dot{\mathbb{P}}^{\delta}$, then $\mathbb{P}_{\delta}$ is $\delta$-c.c., $\left|\mathbb{P}_{\delta}\right| \leq \delta$, and $\vdash_{\mathbb{P}_{\delta}}$ " $\dot{\mathbb{P}}^{\delta}$ is $\prec \delta$-strategically closed" $\left({ }^{7}\right)$. By 2, Facts 1.1 and 1.2], this means that $\nabla_{\delta}$ is preserved from $V$ to $V^{\mathbb{P}^{*}}$, so $V^{\mathbb{P}^{*}} \vDash " \nabla_{\delta}$ holds for every successor and Mahlo cardinal $\delta$ ". Hence, $V^{\mathbb{P}^{*}}$ satisfies properties (1)-(3) of Theorem 1, together with GCH, level by level equivalence between strong compactness and supercompactness, and the fact that $\mathcal{K}$ is the class of supercompact cardinals.

We complete the proof of Theorem 1 by forcing over $V^{\mathbb{P}^{*}}$ with a partial ordering $\mathbb{P}^{* *} \subseteq V^{\mathbb{P}^{*}}$ which preserves everything mentioned in the last sentence of the preceding paragraph and also adds property (4) of Theorem 1 $\mathbb{P}^{* *}=\operatorname{Add}\left(\aleph_{1}, 1\right) * \dot{\mathbb{W}}$, where $\mathbb{\mathbb { W }}$ is a term for Asperó and Friedman's reverse Easton class iteration $\mathbb{W}$ of $[5]$ for adding a locally defined well-ordering $\mathcal{W}$

$\left({ }^{5}\right)$ An outline of the proof is as follows. By the Lévy-Solovay results 15, this is certainly true if $\delta>\kappa_{0}$. If $\delta=\kappa_{0}$ and $\lambda>\kappa_{0}$ is a regular cardinal, let $j: V \rightarrow M$ be an elementary embedding witnessing the $\lambda$ supercompactness of $\kappa_{0}$ such that $M \vDash " \kappa_{0}$ is not $\lambda$ supercompact". We may then write $j\left(\mathbb{P}^{*}\right)=\mathbb{P}^{*} * \dot{\mathbb{Q}}$, where the first ordinal at which $\dot{\mathbb{Q}}$ is forced to do nontrivial forcing is well above $\lambda$. A standard lifting argument now shows that $V^{\mathbb{P}^{*}} \vDash$ " $\kappa_{0}$ is $\lambda$ supercompact". Finally, if $\delta<\kappa_{0}$ and $V \vDash$ " $\delta$ is $\lambda$ supercompact and $\lambda$ is regular", it follows as in 1 that $\lambda$ is below the least $V$-strong cardinal above $\delta$. If we write $\mathbb{P}^{*}=\mathbb{P}_{\delta} * \dot{\mathbb{P}}^{\delta}$, then $\vdash_{\mathbb{P}_{\delta}}$ "نंग $\delta$ is $\eta$-strategically closed for $\eta$ the least inaccessible cardinal above $\lambda$ ". Therefore, to show that $V^{\mathbb{P}^{*}} \vDash$ " $\delta$ is $\lambda$ supercompact", it suffices to show that $V^{\mathbb{P}_{\delta}} \vDash$ " $\delta$ is $\lambda$ supercompact". If $\left|\mathbb{P}_{\delta}\right|<\delta$, then this follows from the results of [15], and if $\left|\mathbb{P}_{\delta}\right|=\delta$, then this follows from the same argument as when $\delta=\kappa_{0}$.

$\left({ }^{6}\right)$ This argument is analogous to the one found in the proof of Lemma 2.1 of this paper. As such, more details will be given when this lemma is proven. The key facts used are that if $V \vDash " \delta<\lambda$ are such that $\delta$ is $\lambda$ supercompact and $\lambda$ is regular", then $V^{\mathbb{P}^{*}} \vDash " \delta$ is $\lambda$ supercompact" and that $\mathbb{P}^{*}$ admits a closure point at $\aleph_{1}$. The main changes are then that $V_{1}$ in Lemma 2.1 is replaced by $V$, and $\mathbb{P}^{* *}$ in Lemma 2.1 is replaced by $\mathbb{P}^{*}$.

$\left({ }^{7}\right)$ If $\delta \geq \kappa_{0}$, then $\mathbb{P}_{\delta}$ is forcing equivalent to $\mathbb{P}_{\kappa_{0}}$, and $\mathbb{P}^{\delta}$ may be taken as a term for trivial forcing. 
of the universe, i.e., a well-ordering satisfying the conditions mentioned earlier. For the exact definition of $\mathbb{W}$, which is rather involved, we refer readers to [5]. We mention only a few relevant facts from [5], which are as follows.

(1) Forcing with $\mathbb{W}$ (and hence forcing with $\mathbb{P}^{* *}$ ) preserves GCH and all cofinalities.

(2) $\Vdash_{\operatorname{Add}\left(\aleph_{1}, 1\right)}$ "Wi is $\aleph_{2}$-directed closed".

(3) $\mathbb{W}=\mathcal{B} * \dot{\mathcal{C}}$, where both $\mathcal{B}$ and $\mathcal{C}$ are direct limits of proper class reverse Easton iterations.

(4) For each Mahlo cardinal $\delta, \mathbb{P}^{* *}=\operatorname{Add}\left(\aleph_{1}, 1\right) * \mathbb{\mathbb { W }}=\operatorname{Add}\left(\aleph_{1}, 1\right) * \dot{\mathcal{B}}_{\delta} *$ $\dot{\mathcal{B}}^{\delta} * \dot{\mathcal{C}}_{\delta} * \dot{\mathcal{C}}^{\delta}$, where $\Vdash_{\operatorname{Add}\left(\aleph_{1}, 1\right)} " \dot{\mathcal{B}}_{\delta}$ is $\delta$-c.c.", $\Vdash_{\operatorname{Add}\left(\aleph_{1}, 1\right)} "\left|\dot{\mathcal{B}}_{\delta}\right|=\delta$ ",

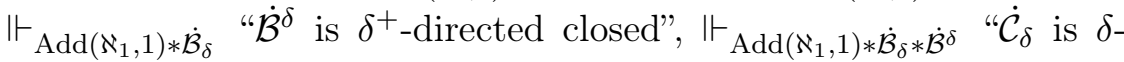

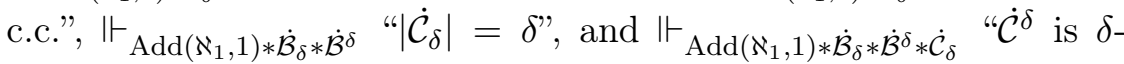
directed closed".

(5) Forcing with $\mathbb{W}$ preserves the $\lambda$ supercompactness of $\delta$, if $\lambda \geq \delta$ are such that $\delta$ is $\lambda$ supercompact and $\lambda$ is regular.

Let $\mathbb{P}=\mathbb{P}^{*} * \dot{\mathbb{P}}^{* *}$. By property (1) above, $V^{\mathbb{P}}=V^{\mathbb{P}^{*} * \dot{\mathbb{P}}^{* *}} \vDash \mathrm{GCH}$.

The following lemma is key to the proof of Theorem 1.

LEMma 2.1. $V^{\mathbb{P}^{*} \mathbb{P}^{* *}}=V^{\mathbb{P}} \vDash$ "Level by level equivalence between strong compactness and supercompactness holds".

Proof. We follow the proof of [1, Lemma 1.3]. Let $V_{1}=V^{\mathbb{P}^{*}}$, and suppose $V_{1}^{\mathbb{P}^{* * *}} \vDash " \kappa<\lambda$ are regular cardinals such that $\kappa$ is $\lambda$ strongly compact and $\kappa$ is not a measurable limit of cardinals $\delta$ which are $\lambda$ supercompact". Suppose $V_{1} \vDash$ " $\delta$ is $\lambda$ supercompact". By the results of [15], $V_{1}^{\operatorname{Add}\left(\aleph_{1}, 1\right)} \vDash$ " $\delta$ is $\lambda$ supercompact", and by property (5) above, $V_{1}^{\operatorname{Add}\left(\aleph_{1}, 1\right) * \mathbb{W}}=V_{1}^{\mathbb{P}^{* *}} \vDash " \delta$ is $\lambda$ supercompact" as well. This means that $V_{1} \vDash$ " $\kappa<\lambda$ are regular cardinals such that $\kappa$ is not a measurable limit of cardinals $\delta$ which are $\lambda$ supercompact".

By property (4) above, $\mathbb{P}^{* *}$ is mild with respect to $\kappa$. Therefore, by the fact $\mathbb{P}^{* *}=\operatorname{Add}\left(\aleph_{1}, 1\right) * \mathbb{W}\left(\right.$ so $\mathbb{P}^{* *}$ admits a closure point at $\left.\aleph_{1}\right)$ and Theorem 2 $V_{1} \vDash$ " $\kappa$ is $\lambda$ strongly compact". Hence, by level by level equivalence between strong compactness and supercompactness in $V_{1}, V_{1} \vDash$ " $\kappa$ is $\lambda$ supercompact". Consequently, as in the previous paragraph, $V_{1}^{\mathbb{P}^{* *}}=V^{\mathbb{P}^{*} * \mathbb{P}^{* *}}=V^{\mathbb{P}} \vDash " \kappa$ is $\lambda$ supercompact" as well. This completes the proof of Lemma 2.1.

As we have just shown, if $V^{\mathbb{P}^{*}} \vDash$ " $\delta<\lambda$ are such that $\delta$ is $\lambda$ supercompact and $\lambda$ is regular", then $V^{\mathbb{P}^{*} \dot{\mathbb{P}}^{* *}}=V^{\mathbb{P}} \vDash$ " $\delta$ is $\lambda$ supercompact". Since $\mathbb{P}^{* *}$ admits a closure point at $\aleph_{1}$, as before, it follows that $V^{\mathbb{P}^{*} * \dot{\mathbb{P}}^{* *}}=V^{\mathbb{P}} \vDash$ " $\mathcal{K}$ is the class of supercompact cardinals (so $\kappa_{0}$ is the least supercompact cardinal)". Therefore, by Lemma 2.1, the proof of Theorem 1 will be complete once we have shown that properties (1)-(4) of its statement hold in $V^{\mathbb{P}}$. 
By the definition of $\mathbb{P}^{* *}$, property (4) of the statement of Theorem 1 holds in $V^{\mathbb{P}^{*} * \dot{\mathbb{P}}^{* *}}=V^{\mathbb{P}}$. Because forcing with $\mathbb{P}^{* *}$ adds a Cohen subset of $\aleph_{1}$ and preserves GCH, as earlier, $V^{\mathbb{P}^{*} * \mathbb{P}^{* *}}=V^{\mathbb{P}} \vDash " \searrow_{\delta}$ holds for every successor cardinal $\delta$ ". For $\delta$ such that $V^{\mathbb{P}^{*} * \mathbb{P}^{* *}}=V^{\mathbb{P}} \vDash " \delta$ is a Mahlo cardinal", it is of course once again the case that $V^{\mathbb{P}^{*}} \vDash$ " $\delta$ is a Mahlo cardinal". By successively applying the factorization of $\mathbb{P}^{* *}$ found in property (4) above and [2, Facts 1.1 and 1.2], we yet again have that $\diamond_{\delta}$ is preserved from $V^{\mathbb{P}^{*}}$ to $V^{\mathbb{P}^{*} * \mathbb{P}^{* *}}=V^{\mathbb{P}}$, so as previously, $V^{\mathbb{P}} \vDash " \nabla_{\delta}$ holds for every successor and Mahlo cardinal $\delta$ ". Thus, property (1) of the statement of Theorem 1 holds in $V^{\mathbb{P}}$.

To show that properties (2) and (3) of the statement of Theorem 1 hold in $V^{\mathbb{P}}$, we begin by assuming that $\delta \in S$. Because both $\square_{\delta}$ and the existence of a gap 1 morass at $\delta$ are upwards absolute to a cofinality preserving generic extension, $V^{\mathbb{P}^{*} * \mathbb{P}^{* *}}=V^{\mathbb{P}} \vDash " \square \square_{\delta}$ holds, and $\delta$ carries a gap 1 morass". Once more, by successively applying the factorization of $\mathbb{P}^{* *}$ given in property (4) above, together with its chain condition and directed closure properties, we may infer that $V^{\mathbb{P}^{*} * \mathbb{P}^{* *}}=V^{\mathbb{P}} \vDash " S$ is a stationary subset of $\kappa_{0}$ ". Thus, property (2) of the statement of Theorem 1 holds in $V^{\mathbb{P}}$. Then, since $\mathbb{P}^{* *}$ admits a closure point at $\aleph_{1}$ and forcing with $\mathbb{P}^{* *}$ preserves cofinalities, we have as earlier that $\operatorname{Safe}(\delta)$ is upwards absolute to $V^{\mathbb{P}^{*} * \dot{\mathbb{P}}^{* *}}=V^{\mathbb{P}}$ for every infinite cardinal $\delta$. By another application of upwards absoluteness, we have as before that $V^{\mathbb{P}^{*} * \dot{\mathbb{P}}^{* *}}=V^{\mathbb{P}} \vDash$ " $\square{ }_{\delta}^{T}$ holds for every infinite cardinal $\delta$, where $T=\operatorname{Safe}(\delta)$ ". Hence, property (3) of the statement of Theorem 1 holds in $V^{\mathbb{P}}$. This completes the proof of Theorem 1 .

We conclude by asking the general question of which additional $L$-like principles are consistent with GCH and level by level equivalence between strong compactness and supercompactness. In particular, as is true of the model constructed in [3], is it possible to infer that $\diamond_{\delta}^{+}$holds for every $\delta$ which is the successor of a regular cardinal in the model of Theorem 11? Is it possible to infer that $\nabla_{\delta}$ holds in this model for any non-Mahlo inaccessible cardinal $\delta$ ? Is it possible to obtain further instances of morasses? By [6, page 644, last paragraph], $\diamond_{\delta}^{+}$for $\delta$ a regular cardinal is destroyed by forcing with $\operatorname{Add}\left(\delta, \delta^{+}\right)$(the standard partial ordering for adding $\delta^{+}$many Cohen subsets of $\delta$ ). In addition, the preservation theorems for $\nabla_{\delta}$ when $\delta$ is inaccessible seem to require a chain condition which is automatically true only when $\delta$ is also a Mahlo cardinal. It is therefore unclear at the moment which partial orderings preserve the various forms of $\diamond$.

Acknowledgments. The author wishes to thank the referee for helpful comments, suggestions, and corrections which have been incorporated into the current version of the paper.

The author's research was partially supported by PSC-CUNY grants and CUNY Collaborative Incentive grants. 


\section{References}

[1] A. Apter, Diamond, square, and level by level equivalence, Arch. Math. Logic 44 (2005), 387-395.

[2] - Stationary reflection and level by level equivalence, Colloq. Math. 115 (2009), $113-128$.

[3] A. Apter and J. Cummings, An L-like model containing very large cardinals, Arch. Math. Logic 47 (2008), 65-78.

[4] A. Apter and S. Shelah, On the strong equality between supercompactness and strong compactness, Trans. Amer. Math. Soc. 349 (1997), 103-128.

[5] D. Asperó and S. Friedman, Large cardinals and locally defined well-orders of the universe, Ann. Pure Appl. Logic 157 (2009), 1-15.

[6] A. Brooke-Taylor, Large cardinals and definable well-orders on the universe, J. Symbolic Logic 74 (2009), 641-654.

[7] A. Brooke-Taylor and S. Friedman, Large cardinals and gap-1 morasses, Ann. Pure Appl. Logic 159 (2009), 71-99.

[8] J. Cummings and E. Schimmerling, Indexed squares, Israel J. Math. 131 (2002), $61-99$.

[9] M. Foreman and M. Magidor, A very weak square principle, J. Symbolic Logic 62 (1997), 175-196.

[10] S. Friedman, Forcing condensation, submitted for publication.

[11] —, Large cardinals and L-like universes, in: Set Theory: Recent Trends and Applications, A. Andretta (ed.), Quad. Mat. 17, Seconda Univ. Napoli, 2006, 93-110.

[12] J. D. Hamkins, Extensions with the approximation and cover properties have no new large cardinals, Fund. Math. 180 (2003), 257-277.

[13] —, Gap forcing, Israel J. Math. 125 (2001), 237-252.

[14] -, Gap forcing: generalizing the Lévy-Solovay theorem, Bull. Symbolic Logic 5 (1999), 264-272.

[15] A. Lévy and R. Solovay, Measurable cardinals and the continuum hypothesis, Israel J. Math. 5 (1967), 234-248.

[16] T. Menas, On strong compactness and supercompactness, Ann. Math. Logic 7 (1974), 327-359.

[17] S. Shelah, Diamonds, submitted for publication.

Arthur W. Apter

Department of Mathematics

Baruch College of CUNY

New York, NY 10010, U.S.A.

and

The CUNY Graduate Center, Mathematics

365 Fifth Avenue

New York, NY 10016, U.S.A.

E-mail: awapter@alum.mit.edu

http://faculty.baruch.cuny.edu/apter

Received January 2, 2009;

received in final form March 9, 2009 